\title{
Efficacy of a Topical Formulation of Sodium Bicarbonate in Mild to Moderate Stable Plaque Psoriasis: a Randomized, Blinded, Intrapatient, Controlled Study
}

\author{
Vittorio Mazzarello - Gabriella Piu - Marco Ferrari (D) · \\ Giorgio Piga
}

Received: February 6, 2019 / Published online: May 10, 2019 (C) The Author(s) 2019

\section{ABSTRACT}

Introduction: Psoriasis is a chronic inflammatory disease characterized by the presence of erythematosquamous lesions. A wide variety of topical treatments for therapy of this pathology are available, including sodium bicarbonate (SB). A few papers reported in literature focus on use of SB baths for treatment of psoriasis, but none assess evidence concerning the efficacy of SB topical preparations. This study aimed to determine the effectiveness of a galenic SB in lanette vax formulation compared with lanette vax base in mild to moderate stable plaque psoriasis.

Methods: A randomized, double-blind, intrapatient, controlled study was performed in 28 days. Thirty patients of both genders were selected for testing. A blinded investigator evaluated the patients' psoriasis using a modified Psoriasis Area and Severity Index (PASI), body surface area (BSA), and objective parameters using sensors (Multiprobe Adapter MPA5; Courage \& Khazaka Electronic $\mathrm{GmbH}$, Cologne, Germany).

Enhanced digital features To view enhanced digital features for this article go to https://doi.org/10.6084/ m9.figshare.8048048.

V. Mazzarello · G. Piu · M. Ferrari ( $₫)$ · G. Piga

Skinlab, Department of Biomedical Sciences,

University of Sassari, Sassari, Italy

e-mail: dr.marcoferrari@gmail.com
Results: Data analysis of objective parameters highlighted that use of the SB topical preparation led to no improvement in skin hydration, no reduction in transepidermal water loss, and no decrease of erythema. The modified PASI and BSA did not change from baseline.

Conclusions: The results obtained show that use of the studied product did not improve psoriatic lesions.

Keywords: Emollient; Psoriasis; Sodium bicarbonate; Topical treatment

\section{INTRODUCTION}

Psoriasis vulgaris is a common skin disorder characterized by focal formation of inflamed, raised plaques that constantly shed scales derived from excessive growth of skin epithelial cells [1]. The common form of psoriasis is plaque psoriasis, in which patients may have sharply circumscribed, round oval, or nummular plaques [2]. In most cases, the severity of this disease is mild as limited body surface area is affected [3]. Mild psoriasis can often be treated with topical agents, although adherence to topical therapy is often suboptimal. Patient satisfaction with topical therapies is low due to frequent relapses, because treatments can be greasy, sticky, and malodorous in application, and can cause staining of clothing and linens $[4,5]$. For this reason, some psoriatic patients 
constantly seek, especially on the Internet and online forums, new therapies using new or old chemical compounds having antipsoriatic activity; among others, use of sodium bicarbonate (SB) compounds has been suggested. SB or baking soda is the monosodium salt of carbonic acid; its therapeutic properties have been widely studied to demonstrate its effectiveness against several pathologies. In particular, for skin pathologies, it can be employed as an antimicrobial and for treatment of aquagenic pruritus $[6,7]$. In relation to psoriasis, therapy with SB has been investigated in balneotherapy $[8,9]$. Patients subjected to these treatments showed a significant improvement in psoriatic lesions as well as quality of life. However, topical application of SB is an anecdotal remedy, and controlled studies are lacking. The aim of this study is to evaluate whether application of an SB topical product can improve psoriatic lesions, also when performed outside of a comfortable environment such as balneotherapy.

\section{METHODS}

\section{Product}

In this study, a galenic SB (Sigma-Aldrich) 30\% in lanette wax $N$ (BASF Care Creation) (INCI: cetearyl alcohol, sodium cetearyl sulfate) formulation was used $[10,11]$.

\section{Study Design}

This single-center, randomized, double-blinded, intraindividual, comparative study enrolled 30 adult patients of both genders. The study was conducted at the Skinlab, Department of Biomedical Sciences, University of Sassari, Italy. All clinical studies were reviewed and approved by the Ethical Committee Local Health Unit 1 (Sassari, Italy). Inclusion criteria were age between 20 and 60 years and symmetric, stable plaque psoriasis on arms, legs, and some patches on the trunk with maximum Psoriasis Area and Severity Index (PASI) score of 10.8. Patients were excluded if they were underage, pregnant or breastfeeding women, had received topical or systemic treatments during the 3 months prior to experiments, or had positive anamnesis for allergic contact dermatitis. Treatment was performed randomly on the left and right side of the body, applying the SB in lanette vax formulation on one side and lanette vax on the other side, while patches on the trunk were not treated. During the first clinical visit to standardize the dose of the two products, which is different for each patient as it is linked to the extension of the patches, we used a Silkidose ${ }^{\circledR}$ (Galderma Laboratories) to indicate to each patient the quantity to apply on the areas to be treated. The patients were instructed to apply the medications twice daily for 28 days with a slight massage until absorption and were prohibited from using any emollient during application. Treatment efficacy was assessed by a blinded investigator based on noninvasive skin analysis and the disease clinical progression indexes-PASI and body surface area (BSA). Before starting the experiment, for noninvasive analysis, three skin reference areas (elbow, knee, and trunk) for each patient were selected: (a) a psoriatic patch treated with galenic SB, (b) a second contralateral psoriatic patch treated with lanette wax $N$ (vehicle), and (c) a third psoriatic patch of the trunk not treated, as negative control. Objective measurements were made on these patches. The skin barrier function was evaluated by measuring the transepidermal water loss (TEWL) using a Tewameter ${ }^{\mathrm{TM}}$ 300 (Courage + Khazaka Electronic GmbH, Cologne, Germany), and hydration in the stratum corneum was assessed using a Corneometer CM 825 (Courage + Khazaka Electronic GmbH, Cologne, Germany). Skin color was measured using a Mexameter MX 16 (Courage + Khazaka Electronic GmbH, Cologne, Germany). An erythema index was computed from the intensity of the absorbed and reflected light at 568 and $660 \mathrm{~nm}$, respectively [12]. These devices, specifically the corneometer and tewameter, have already been used to evaluate psoriatic skin and the efficacy of moisturizing [13]. Few and contradictory studies have measured the skin $\mathrm{pH}$ of psoriatic lesions; the authors hypothesized that significantly lower skin $\mathrm{pH}$ in psoriatic skin compared with healthy subjects 
could be a cause or consequence of excess fatty acids [14]. Instrumental assessments were performed at time to (baseline) and after $60 \mathrm{~min}$ (t60m) during the short-term test. During the long-term tests, patients were evaluated after 7 days $(t 7 \mathrm{~d}), 14$ days $(t 14 \mathrm{~d})$, and 28 days $(t 28 \mathrm{~d})$ of continuous application of the product. At each phase of the research, each volunteer was asked not to clean test zones for at least $12 \mathrm{~h}$ prior to examination. Before measurements, patients remained for $30 \mathrm{~min}$ in an air-conditioned room where the assessment was going to take place with skin reference areas uncovered, to favor acclimatization of the skin to room temperature and humidity conditions. All measurements were performed on central areas of selected patches by the same experimenter. The PASI score was calculated at baseline, $t 7 \mathrm{~d}$, $t 14 \mathrm{~d}$, and the end of the treatment period. Because this was an intrapatient study, we used a modified PASI that included the upper and lower extremities and trunk only. Patients scored their psoriasis symptoms, including erythema, infiltration, and desquamation, for the test areas (skin surface extremities of the left and right side and skin of the trunk). The sum of the scores was calculated for each parameter, using a similar scoring system to that of the original PASI score, viz. no sign $=0$, slight $=1$, moderate $=2$, severe $=3$ [15]. As only patients with slight to moderate severity of psoriasis were included, the maximum score for each test area was 3.6. The difference between the score sums on the test area was not allowed to be more than 0.50. The local PASI score was evaluated at each visit during the long-term test. The primary efficacy endpoint was the difference in the percent change in the baseline PASI score between the three test areas at week 4. BSA was assessed for a single-area test at each visit during the long-term test.

\section{Statistical Analysis}

All results were compared with each baseline measurement by paired $t$ tests, or with a nonparametric test (Wilcoxon signed-rank test) when difference means did not have normality characteristics, as verified by Shapiro-Wilk test.
All data analyses were performed using Stata SE 15.0 with $p<0.05$ considered as statistically significant. All data are expressed as mean $\pm s$ tandard deviation (SD), and beyond measured values, differences versus baseline are reported.

\section{Compliance with Ethical Guidelines}

All procedures performed in studies involving human participants were in accordance with IntegReview IRB, who granted an exemption from Institutional Review Board oversight in accordance with 45 CFR 46.101(b) and with the 1964 Helsinki Declaration and its later amendments or comparable ethical standards. Informed consent was obtained from all individual participants included in the study.

\section{RESULTS}

All subjects completed the study. Their demographic characteristics are reported in Table 1. Baseline disease characteristics were also comparable across the treatment sides (Table 2), and the mean target lesion size was in the range of $12-94 \mathrm{~cm}^{2}$. All procedures and clinical analysis were performed under identical environmental conditions, at mean temperature of $22.1 \pm 1.7^{\circ} \mathrm{C}$ and mean humidity of $55 \pm 4 \%$, between 9 a.m. and 2 p.m.

Table 1 Demographics of volunteers enrolled in the study Demographic characteristic of patients

\begin{tabular}{ll}
\hline & $\boldsymbol{N}=\mathbf{3 0}$ \\
\hline Dropout & $N=0$ \\
Male & $20(64 \%)$ \\
Female & $10(33 \%)$ \\
Age (range) & $26-52$ years \\
Age (mean \pm SD) & $36.4 \pm 9.31$ years \\
\hline
\end{tabular}


Table 2 Summary of baseline patient disease characteristics, mean $\pm \mathrm{SD}$

\begin{tabular}{llll}
\hline $\begin{array}{l}\text { Disease } \\
\text { characteristic }\end{array}$ & SB & Vehicle & Control \\
\hline $\begin{array}{l}\text { Modified } \\
\text { PASI score }\end{array}$ & $3.42 \pm 0.36$ & $3.23 \pm 0.46$ & $2.82 \pm 0.59$ \\
$\begin{array}{l}\% \text { BSA } \\
\text { affected }\end{array}$ & $2.4 \pm 1.8$ & $2.5 \pm 1.7$ & $1.9 \pm 1.2$ \\
$\begin{array}{c}\text { Size of target } \\
\text { lesion }\left(\mathrm{cm}^{2}\right)\end{array}$ & $49.7 \pm 32.8$ & $52.1 \pm 42.6$ & $24.6 \pm 20.1$ \\
\hline
\end{tabular}

$S B$ random extremities area treated with galenic sodium bicarbonate $30 \%$ in lanette wax $N$, Vehicle contralateral random extremities area treated only with lanette wax $N$, Control trunk area not treated

\section{Efficacy Results: Noninvasive Skin Analysis}

Hydration values of psoriatic patches significantly increased compared with baseline only at 60 min after SB application. Psoriatic patches treated with vehicle showed a similar increase of skin hydration values compared with baseline. Comparisons between these two groups did not show statistically significant differences $(p=0527)$. SB cream applied on psoriatic patches did not modify TEWL and erythema values, in a similar way to vehicle, during shortand long-term tests (Table 3).

\section{Efficacy Results: PASI and BSA}

The change in modified PASI from baseline is presented in Table 4 . At day 28, there was a $5.7 \%$ decrease in PASI for the SB test area, but as for the BSA score, no significant improvement of psoriatic lesions.

\section{DISCUSSION}

SB is widely used in medicine to treat numerous diseases, with several applications in the field of internal medicine; in fact, its efficacy when used on a topical basis has been demonstrated in many studies, for instance in treatment of gingivitis [16], oral hygiene protection [17], as well as associated with paracetamol in pharyngitis

Table 3 Descriptive statistics, mean $\pm \mathrm{SD}$, and $p$ value for all observed variables

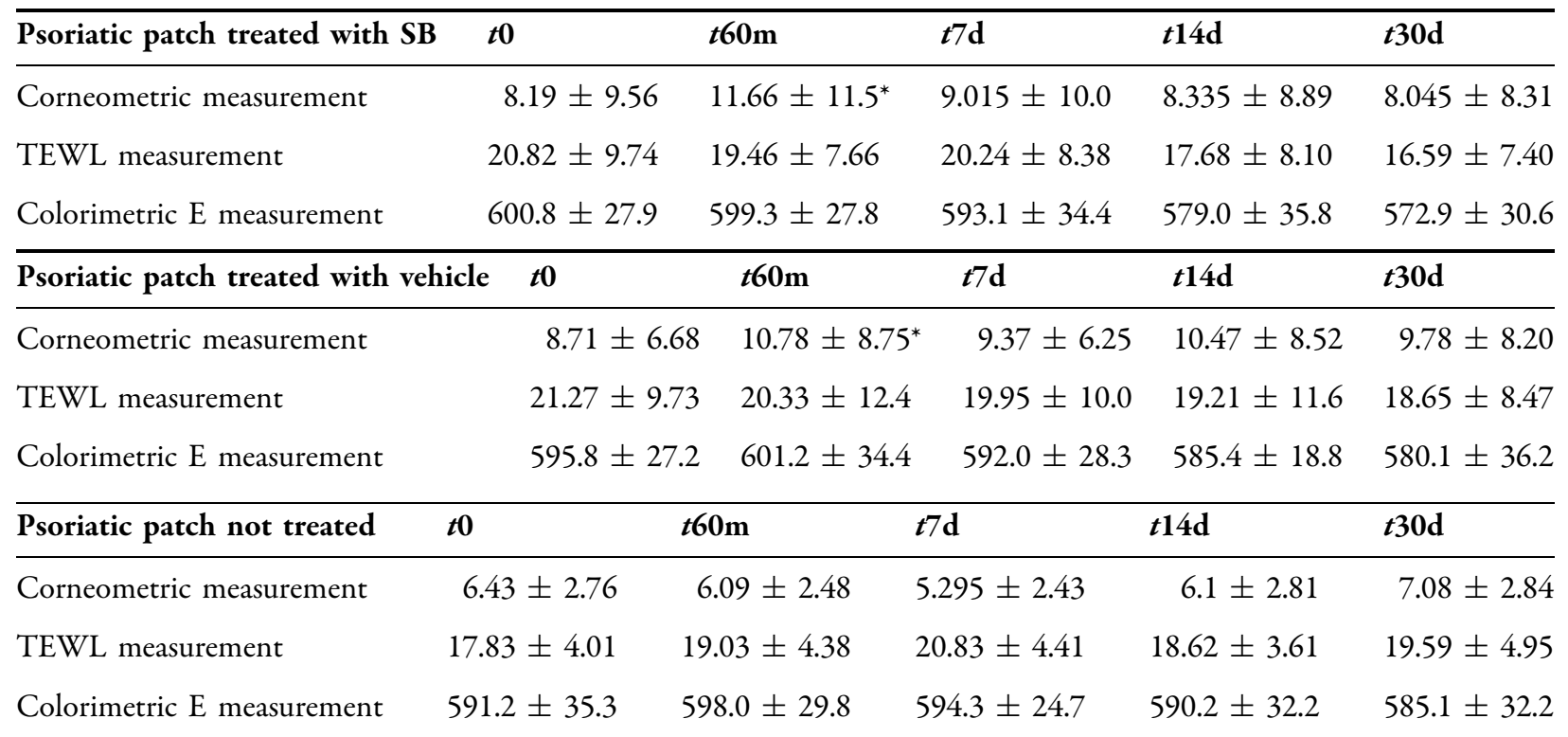

$S B$ galenic sodium bicarbonate $30 \%$ in lanette wax $N$, Vebicle only lanette wax $N$ ${ }^{*} p<0.05$ 
Table 4 Change of PASI from baseline at each visit, $N(\%)$

\begin{tabular}{llcc}
\hline Modified PASI score & SB & Vehicle & Control \\
\hline$t 7 \mathrm{~d}$ & $-0.07(-2.0 \%)$ & $-0.13(-1.3 \%)$ & $-0.18(-2.6 \%)$ \\
$t 14 \mathrm{~d}$ & $-0.1(-4.0 \%)$ & $-0.2(-4.3 \%)$ & $0.05(5.21 \%)$ \\
$t 28 \mathrm{~d}$ & $-0.2(-5.7 \%)$ & $-0.2(-4.0 \%)$ & $-0.12(-1.0 \%)$ \\
\hline
\end{tabular}

$S B$ random extremities area treated with galenic sodium bicarbonate $30 \%$ in lanette wax $N$, Vehicle contralateral random extremities area treated only with lanette wax $N$, Control trunk area not treated

and in interstitial cystitis [18, 19]. In vitro antimicrobial efficacy against microorganisms such as dermatophytes (Candida albicans, Escherichia coli, Staphylococcus aureus, group B streptococci) has also been studied [20, 21]. However, for treatment of psoriasis, the only form of SB whose effectiveness has been demonstrated is balneotherapy. Indeed, in literature, after getting wet in a water solution with 350-500 $\mathrm{g}$ dissolved SB on alternate days for 3 weeks, 19 subjects showed a significant improvement in psoriatic lesions and, at the same time, quality of life, unlike the placebo group [10]. Another study might confirm these properties: 30 patients affected by psoriasis were treated at Castellammare of Stabia spa (Napoli, Italy) with mud and baths containing water rich in chloride-sulfide-bicarbonate, coming from mineral springs, showing an improvement in PASI and itchy symptoms after a 12-day treatment [9]. The cause of this improvement is not clear, so far. It has been hypothesized that the most important factor is the basic $\mathrm{pH}$ created by SB dissolved in water, which should have a keratolytic action or play a role in activating some molecules involved in the disease or exert an action on the skin microbiome [22]. As the skin microbiome is typically altered in psoriasis [23], some authors showed that psoriatic subjects treated with La Roche-Posay spa water, rich in selenium, had a net improvement in lesions due to changes induced in the microbiome by this water [24]. As far as the work carried out by Costantino et al. is concerned, the favorable environment of the spas that may have contributed to the clinical improvement of lesions should also be taken into account. Besides, the efficacy of balneotherapy has been well described by several authors [25-27]. However, in these studies, it is not clear how much the psychological benefits associated with balneotherapy affected the improvement of lesions and how much the changes were actually caused by the action of the thermal water. Certainly, these two effects act synergistically, thus causing the regression of cutaneous inflammation. Topical use of SB in other formulations for psoriasis treatment is thus still anecdotal, and there are no studies proving its effectiveness. In some books or on social forums, its use in galenic formulations with castor oil or olive oil is recommended as a natural treatment for considerably thick psoriatic lesions [28]. The aim of this study is to evaluate the efficacy of SB in a galenic preparation for improving psoriatic lesions. It seemed important to our team to evaluate whether SB is effective even when used independently of balneotherapy performed at a spa or home. Our study aimed to evaluate the ability of a topical $\mathrm{SB}$ product to improve cutaneous lesions in subjects affected by psoriasis, but the results obtained in this study should be considered negative. They do not confirm the efficacy of topical SB cream in improving stable psoriatic plaques, untreated with other therapies for at least 3 months. In fact, it was not possible to highlight any improvement of PASI, BSA, erythema, hydration, or TEWL after 28 days of daily treatment. The patches treated with SB, patches treated with the base, and patches not treated remained stable throughout the treatment, and no statistically significant differences were found between the groups analyzed. This lack of response could be linked to the fact that the formulation did not maintain the basic $\mathrm{pH}$ after application or the concentration used was insufficient to obtain the expected results. 


\section{Limitations}

This study was conducted on a limited sample of 30 individuals affected by stable plaques of psoriasis vulgaris. To evaluate the results, we used nonparametric tests with post hoc analysis. Such well-designed small studies are valuable but must be interpreted carefully, and data should be used to design larger confirmatory studies. Therefore, this is an exploratory work that offers some important indications for exploration in further studies.

\section{CONCLUSIONS}

The 30\% SB product tested in this study did not have the ability to improve typical aspects of psoriatic patches after just 1 month of use. However, it should be considered that, due to the small size of the experimental sample considered, further studies are warranted to confirm these results.

\section{ACKNOWLEDGEMENTS}

We thank the participants of the study.

Funding. No funding or sponsorship was received for this study or publication of this article. Article processing charges were funded by the authors.

Authorship. All named authors meet the International Committee of Medical Journal Editors (ICMJE) criteria for authorship for this article, take responsibility for the integrity of the work as a whole, and have given their approval for this version to be published.

Disclosures. Vittorio Mazzarello, Gabriella Piu, Marco Ferrari and Giorgio Piga have nothing to disclose.

Compliance with Ethics Guidelines. All procedures performed in studies involving human participants were in accordance with IntegReview IRB, who granted an exemption from Institutional Review Board oversight in accordance with 45 CFR 46.101(b) and with the 1964 Helsinki declaration and its later amendments or comparable ethical standards. Informed consent was obtained from all individual participants included in the study.

Data Availability. The datasets generated during and/or analyzed during the current study are available from the corresponding author on reasonable request.

Open Access. This article is distributed under the terms of the Creative Commons Attribution-NonCommercial 4.0 International License (http://creativecommons.org/licenses/ by-nc/4.0/), which permits any noncommercial use, distribution, and reproduction in any medium, provided you give appropriate credit to the original author(s) and the source, provide a link to the Creative Commons license, and indicate if changes were made.

\section{REFERENCES}

1. Krueger J, Bowcock A. Psoriasis pathophysiology: current concepts of pathogenesis. Ann Rheum Dis. 2005;64:30-6.

2. Langley RGB, Krueger GG, Griffiths CEM. Psoriasis: epidemiology, clinical features, and quality of life. Ann Rheum Dis. 2005;64:18-23.

3. Sujay K, Kanika S. An open label prospective randomized trial to compare the efficacy of coal tarsalicylic acid ointment versus calcipotriol/betamethasone dipropionate ointment in the treatment of limited chronic plaque psoriasis. Indian J Dermatol. 2014;59:579-83.

4. Devaux S, Castela A, Archier E, Gallini A, Joly P, Misery L, Paul C. Adherence to topical treatment in psoriasis: a systematic literature review. J Eur Acad Dermatol Venereol. 2012;26:61-7.

5. Callis Duffin K, Yeung H, Takeshita J, Krueger GG, Robertson AD, Troxel AB, Gelfand JM. Patient satisfaction with treatments for moderate-to-severe plaque psoriasis in clinical practice. Br J Dermatol. 2014;170:672-80.

6. Bircher AJ. Aquagenic pruritus: treatment with sodium bicarbonate and evidence for a seasonal form. J Am Acad Dermatol. 1989;21:817. 
7. Wolf R, Krakowski A. Variations in aquagenic pruritus and treatment alternatives. J Am Acad Dermatol. 1988;18:1081-3.

8. Costantino M, Lampa E. Psoriasis and mud bath therapy: clinical-experimental study. Clin Ter. 2005; 156:145-9.

9. Verdolini R, Bugatti L, Filosa G, Mannello B, Lawlor $\mathrm{F}$, Cerio RR. Old fashioned sodium bicarbonate baths for the treatment of psoriasis in the era of futuristic biologics: an old ally to be rescued. J Dermatol Treat. 2005;16(1):26-30.

10. Van der Rhee HJ, Polano MK. Treatment of psoriasis vulgaris with a low-dosage Ro 10-9359 (Tigason) orally combined with corticosteroids topically. In: Orfanos CE, editor. Retinoids. Berlin: Springer; 1981. https://doi.org/10.1007/978-3-642-68023-6_ 25.

11. Arnold WP, Glade CP, Mier PD, van de Kerkhof PC. Effects of sphingosine, isoquinoline and tannic acid on the human tape-stripping model and the psoriatic lesion. Skin Pharmacol. 1993;6:193-9.

12. Clarys P, Alewaeters K, Lambrecht R, et al. Skin color measurements: comparison between three instruments: the Chromameter $^{\circledR}$, the Derma Specrometer $^{\circledR}$, and the Mexameter ${ }^{\circledR}$. Skin Res Technol. 2000;6:230-8.

13. Rim JH, Jo SJ, Park JY, Park BD, Youn JI. Electrical measurement of moisturizing effect on skin hydration and barrier function in psoriasis patients. Clin Exp Dermatol. 2005;30:409-13.

14. Bigliardi PL. Role of skin $\mathrm{pH}$ in psoriasis, $\mathrm{pH}$ of the skin: issues and challenges. Curr Probl Dermatol. 2018;54:108-14 (Basel, Karger).

15. Thompson M, Feutren G. Psoriasis area and severity index. Basel: Novartis Pharma AG; 1997.

16. Lomax A, Patel S, Wang N, Kakar K, Kakar A, Bosma ML. A randomized controlled trial evaluating the efficacy of a $67 \%$ sodium bicarbonate toothpaste on gingivitis. Int J Dent Hyg. 2016;15(4):e35-41.

17. Zambon JJ, Mather ML, Gonzales Y. A microbiological and clinical study of the safety and efficacy of baking-soda dentifrices. Compend Contin Educ Dent Suppl. 1996;17(19):S39-44.
18. Burnett I, Schacht B, Sanner K, Bey M, Grattan T, Littlejohn S. Onset of analgesia of a paracetamol tablet containing sodium bicarbonate: a doubleblind, placebo-controlled study in adult patients with acute sore throat. Clin Ther. 2006;28:1273-8.

19. Welk BK, Teichman JM. Dyspareunia response in patients with interstitial cystitis treated with intravescical lidocaine, bicarbonate, and heparin. Urology. 2008;71:67-70.

20. Letscher-Bru V, Obszynski CM, Samsoen M, Sabou M, Waller J, Candolfi E. Antifungal activity of sodium bicarbonate against fungal agents causing superficial infections. Mycopathologia. 2013;175:153-8.

21. Bidra AS, Tarrand JJ, Roberts DB, Rolston KV, Chambers MS. Antimicrobial efficacy of oral topic agents on microorganism associated with radiated head and neck cancer patients: an in vitro study. Quintessence Int. 2011;42:307-15.

22. Milstone LM. Scaly skin and bath $\mathrm{pH}$ : rediscovering baking soda. J Am Acad Dermatol. 2010;62:885-6.

23. Weyrich LS, Dixit S, Farrer AG, Cooper AJ, Cooper AJ. The skin microbiome: associations between altered microbial communities and disease. Australas J Dermatol. 2015;56:268-74.

24. Martin R, Henley JB, Sarrazin P, Seitè S. Skin microbiome in patients with psoriasis before and after balneotherapy at the thermal care center of La Roche-Posay. J Drugs Dermatol. 2015;14:1400-5.

25. Zumiani G, Tasin L, Urbani F, Tinozzi CC, Carabelli A, Cristofolini M. Clinico-statistical study on hydropinic and balneothermal therapy of psoriatic patients using the low-mineral content waters of the Comano springs. Minerva Med. $1986 ; 77: 627-34$.

26. Riyaz N, Arakkal FR. Spa therapy in dermatology. Indian J Dermatol Venereol Leprol. 2011;77:128-34.

27. Matz H, Orion E, Wolf R. Balneotherapy in dermatology. Dermatol Ther. 2003;16:132-40.

28. Pagano OAJ. Healing psoriasis: the natural alternative. 1st ed. New York: Wiley; 2008. 